Original Paper http://ajol.info/index.php/ijbcs http://indexmedicus.afro.who.int

\title{
Caractérisation physico-chimique et contamination métallique des eaux usées déversées au niveau de la baie de Hann (Dakar/Sénégal)
}

\author{
Ibrahima DIAGNE, Samba DRAME, Momar NDIAYE*, Birame NDIAYE et \\ Abdoulaye DIOP
}

Faculte des sciences et techniques, Laboratoire de Chimie Physique Organique et d'Analyse Environnementale (LCPOAE)-UCAD, Dakar, Sénégal.

"Auteur correspondant ; E-mail: momndiaye@hotmail.com

\section{RESUME}

$\mathrm{Au}$ Sénégal, les eaux usées domestiques et industrielles sont versées directement dans le milieu aquatique sans aucun traitement préalable, pouvant créer une dégradation du milieu marin. Dans le présent travail, nous avons déterminé les paramètres physico-chimiques et métalliques des eaux usées du canal Est, qui débouche sur la baie de Hann, pour vérifier les normes de rejets avant le déversement dans le milieu récepteur. Le canal, situé le long de la côte dakaroise, a été choisi à cause des nombreuses activités qui se déroulent sur la baie de Hann. Les paramètres physiques ( $\mathrm{pH}$, température et conductivité) ont été mesurés in situ à l'aide d'un appareil HANNA instruments $\mathrm{pH} /$ conductivity HI 98129. Les paramètres chimiques et métalliques (ammonium $\left(\mathrm{NH}_{4}{ }^{+}\right)$, chrome $6\left(\mathrm{Cr}^{6+}\right)$, chlorures $\left(\mathrm{Cl}^{-}\right)$, fluorures $\left(\mathrm{F}^{-}\right)$, potassium $\left(\mathrm{K}^{+}\right)$, nitrates $\left(\mathrm{NO}_{3}{ }^{-}\right)$, phosphates $\left(\mathrm{PO}_{4}{ }^{3-}\right)$ et sulfates $\left.\left(\mathrm{SO}_{4}{ }^{2-}\right)\right)$ ont été dosés par un photomètre $\mathrm{PF}-11$. Les résultats obtenus montrent que les paramètres physiques étudiés ne dépassent pas les normes de rejets d'eaux usées dans ce milieu récepteur. Les ions $\mathrm{NO}_{3}{ }^{-}$ n'ont pas été détectés au niveau des échantillons d'eaux usées prélevés alors que les teneurs des éléments $\mathrm{Cr}^{6+}$, $\mathrm{SO}_{4}^{2-}, \mathrm{F}^{-}$et $\mathrm{Cl}^{-}$sont inférieures à la limite de rejets directs dans le milieu récepteur. Cependant les concentrations enregistrées en $\mathrm{NH}_{4}^{+}, \mathrm{K}^{+}$et $\mathrm{PO}_{4}{ }^{3-}$ sont nettement supérieures à la norme fixée par la législation de rejets dans le milieu récepteur. Ainsi, le rejet de ces eaux usées dans ce milieu aquatique peut entrainer l'eutrophisation mais également la raréfaction ou la mort des poissons.

(C) 2017 International Formulae Group. All rights reserved.

Mots clés: Eaux usées, analyses physicochimiques, photométrie, contamination.

\section{Physico-chemical characterization and metallic contamination of wastewater discharged at Hann Bay (Dakar / Senegal)}

\begin{abstract}
In Senegal, domestic and industrial worn water is directly versed in the aquatic environment without any preliminary treatment. That can have as a consequence the degradation of the marine environment. In this work, we characterized some physicochemical and metal parameters the water used before discharge in the receiving medium to check their standards of rejections. For that, the channel of bay of Hann was selected along the coast dakaroise because of many activities which proceed there. The physical parameters $(\mathrm{pH}$, temperature and conductivity) were measured in situ using an apparatus HANNA instruments $\mathrm{pH} /$ conductivity HI 98129. The chemical and metal parameters (ammonium, chromium 6, chloride, fluoride, potassium, nitrate,
\end{abstract}


phosphate and sulphate) were proportioned by a photometer PF-11. The results obtained show that the studied physical parameters do not exceed the standards of water discharges used in this receiving medium. $\mathrm{NO}_{3}{ }^{-}$is not detected on the level of worn water samples the taken whereas contents of the $\mathrm{Cr}^{6+}$ elements ${ }^{+}, \mathrm{SO}_{4}{ }^{2-}, \mathrm{F}^{-}, \mathrm{Cl}^{-}$are lower than the limit of forward thrusts in the receiving medium. However the concentrations recorded out of $\mathrm{NH} 4^{+}, \mathrm{K}^{+}$and $\mathrm{PO}_{4}{ }^{3-}$ are definitely higher than the standard set by the legislation of rejections in the receiving medium. Consequently, the discharge of this water used in this aquatic environment can lead to eutrophication and rarefaction or the death of fish.

(C) 2017 International Formulae Group. All rights reserved.

Keywords: Worn water, physicochemical analyzes, photometry, contamination.

\section{INTRODUCTION}

L'eau de mer est une ressource indispensable à la vie. Elle mérite une préservation particulière parce qu'elle est très menacée par les activités humaines (Ezziane, 2007). En effet, la croissance démographique accompagnée d'une urbanisation rapide, d'une utilisation intensive d'eaux et un défaut d'épuration de ces eaux usées entrainent de nombreuses perturbations pour les milieux naturels et en particulier, le milieu marin (Mc Kinny, 2002). La pollution, liée à un rejet des eaux usées dans ces zones côtières constitue une menace pour la santé publique, les espèces sauvages ainsi que pour les sources de revenus comme la pêche et le tourisme.

La République du Sénégal est un pays d'Afrique de l'ouest, bordé par l'océan atlantique. Comme dans beaucoup d'autres pays d'Afrique, le manque d'assainissement et de systèmes d'épuration peuvent entrainer des conséquences désastreuses pour la santé des habitants, mais aussi pour l'environnement et l'économie du pays. $\mathrm{Ce}$ manque d'assainissement des lieux de vie conduit à la pollution des eaux (Moussa Moumouni, 2005 ; Mouhamed et al., 2012). Ainsi les rejets (déchets, eaux usées...) représentent une grande partie de la pollution actuellement $\mathrm{du}$ pays, menaçant les réserves en poissons. Ce problème est particulièrement noté dans la Baie de Hann, avec ses $15 \mathrm{~km}$ de long.

L'objectif du présent travail consiste à faire des études physico-chimiques et métalliques des eaux de rejets du canal d'évacuation d'eaux usées de la baie de Hann par la méthode photométrique.

\section{MATERIEL ET METHODES}

\section{Sites de prélèvements et échantillonnage}

La région de Dakar reçoit une forte charge organique et minérale engendrée par les rejets domestiques et industriels. Dans le cadre de cette étude, le canal de la baie de Hann a été choisi le long de la côte dakaroise (Figure 1). Le prélèvement des échantillons a été réalisé en trois campagnes (mars, avril et mai 2015) au niveau des différentes stations. Tous les prélèvements d'eau ont été effectués dans des flacons en polyéthylène.

Les échantillons ont été transportés au laboratoire et conservés entre 2 et $5{ }^{\circ} \mathrm{C}$ (Berho, 2008).

\section{La commune de Hann}

Le littoral de la Baie de Hann est limité à l'Est par le département de Dakar et le canal d'évacuation des eaux usées du port. Le canal d'évacuation des eaux usées de cette commune reçoit les déchets ménagers et ceux de $80 \%$ du potentiel industriel du Sénégal. Il est directement connecté à la baie, qui abrite un quai de pêche, lieu de débarquement des produits halieutiques. Plusieurs familles de la capitale et même des habitants de l'intérieur $\mathrm{du}$ pays viennent s'y approvisionner en poissons.

\section{Mesure des paramètres physiques}

Les paramètres physiques (température, le $\mathrm{pH}$ et la conductivité) ont été mesurés in situ à l'aide d'un appareil HANNA instruments pH/conductivity HI 98129.

Pour la mesure du $\mathrm{pH}$, l'appareil a été étalonné avec des solutions tampons $\mathrm{pH}=7,01$ puis $\mathrm{pH}=4,01$. 
Pour la conductivité, l'appareil a été calibré à l'aide d'une solution étalon HI 7031 (1413 $\mathrm{us} / \mathrm{cm}$ ).

La température s'affiche directement en même temps que l'on mesure le $\mathrm{pH}$ ou la conductivité.

\section{Mesure des paramètres chimiques et métalliques \\ Les paramètres chimiques et} métalliques ont été mesurés par un photomètre PF-11 à cuve ronde. Les analyses, réalisées par le phonomètre, ont été faites en quatre étapes:

\section{Préparation de l'échantillon et le blanc}

Le blanc est constitué soit de l'eau déminéralisée soit de l'échantillon prélevé sans ajout de réactifs.

La préparation de l'échantillon à analyser consiste à ajouter des réactifs dans $5 \mathrm{ou} 10 \mathrm{~mL}$ d'échantillons d'eau prélevés. C'est une méthode d'analyse par ajout dosé. Il est important de respecter le temps de lecture prescrit dans le protocole d'analyses pour assurer une homogénéité de la solution à doser.

\section{Préparation du photomètre}

L'appareil comporte plusieurs méthodes de lecture de la teneur de l'élément à doser: Visocolor, Visocolor Eco ou nanocolor. La méthode, à choisir, dépend de chaque élément et sa mesure s'affiche directement sur l'appareil.

\section{Réglage du zéro de la concentration}

Le zéro de la concentration doit être réglé avant chaque mesure.

\section{Mesure de l'échantillon préparé}

Placer l'échantillon préparé dans la cellule de mesure et lire directement la concentration de l'échantillon affichée sur l'écran de l'appareil en mg/L.

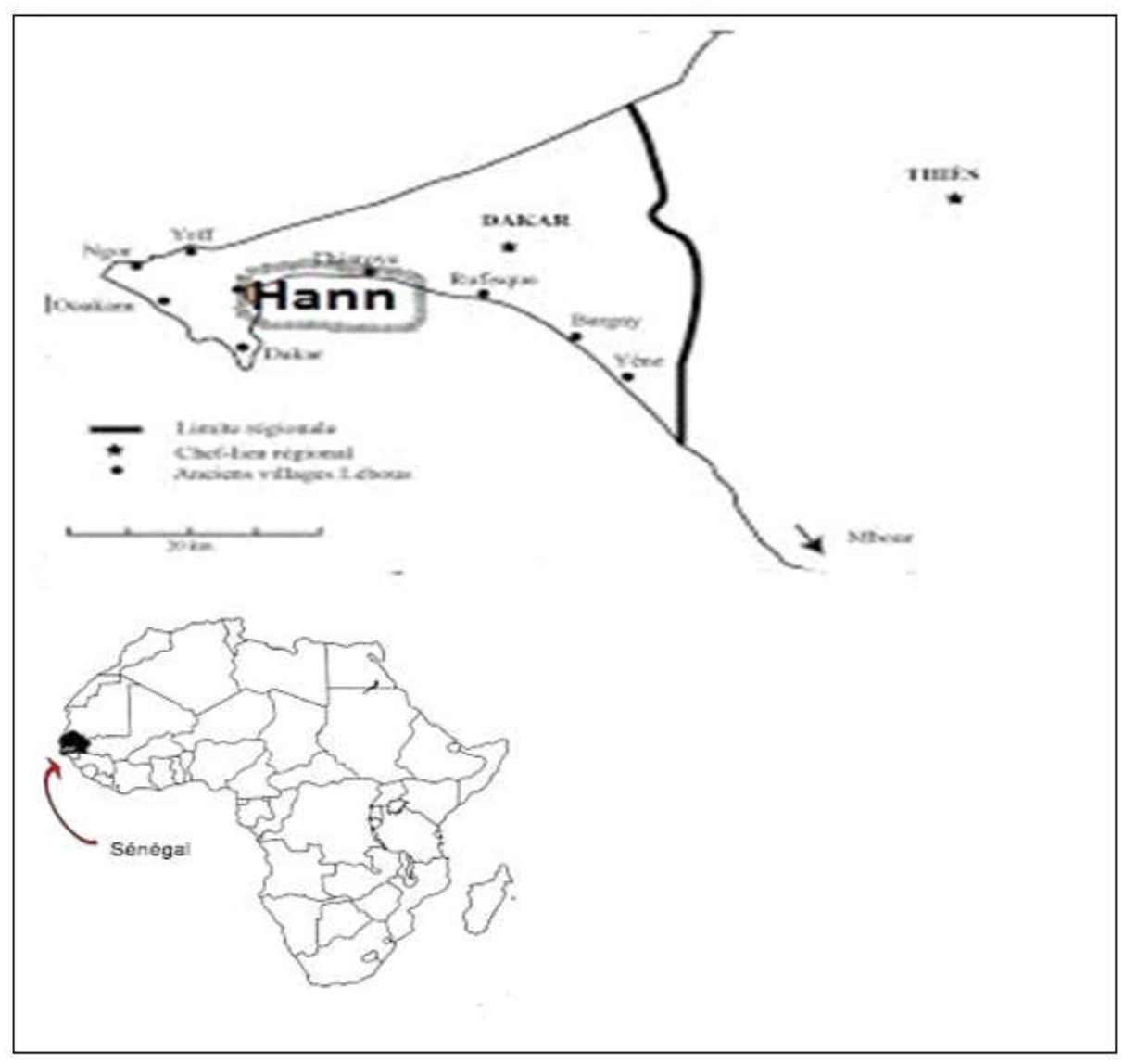

Figure 1: Site de prélèvement des eaux. 


\section{RESULTATS}

\section{Les paramètres physiques}

Les Figures 2,3 et 4 présentent les résultats des mesures $\mathrm{du} \mathrm{pH}$, de la température et de la conductivité, respectivement, des échantillons d'eau prélevés dans les trois sites étudiés.

\section{Les paramètres chimiques et métalliques}

\section{Les cations}

Les Figures 5, 6 et 7 présentent,

respectivement, les résultats de mesures de la teneur des cations $\mathrm{NH}_{4}^{+}$, $\mathrm{K}^{+}$et $\mathrm{Cr}^{6+}$, dans les eaux de rejets, en fonction du temps.

\section{Les anions}

Les Figures 8, 9 et 10 présentent, respectivement, les résultats de mesures de la teneur des anions $\mathrm{Cl}^{-}, \mathrm{PO}_{4}{ }^{3-}$ et $\mathrm{SO}_{4}{ }^{2-}$.

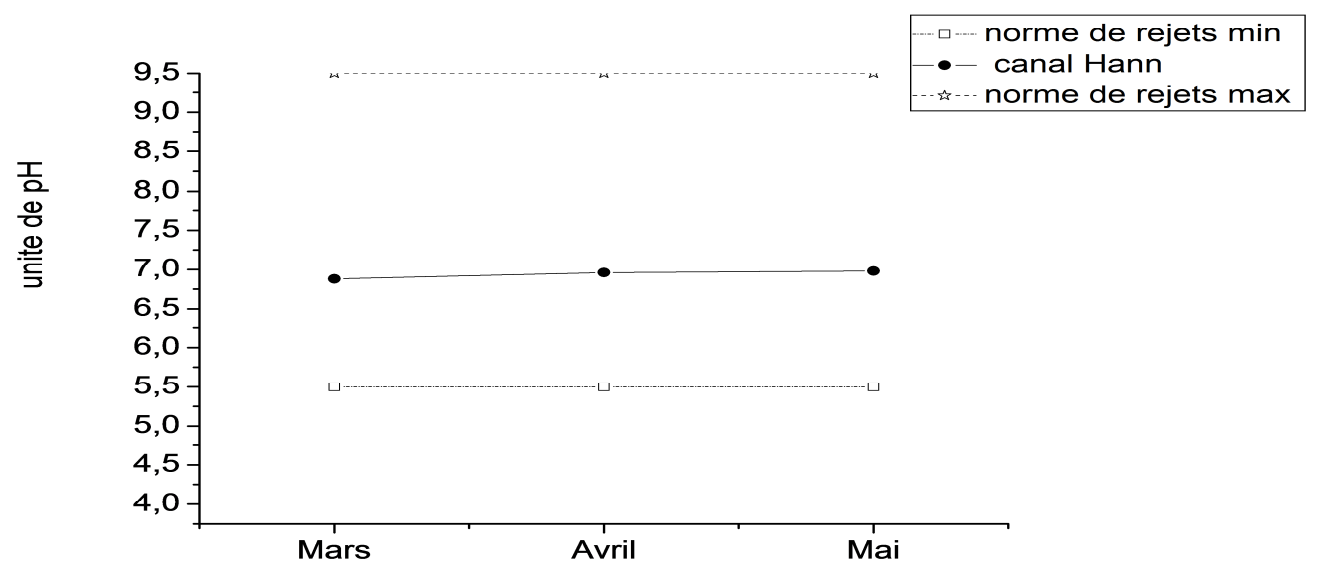

Figure 2: Mesures du pH dans les eaux usées en fonction du temps.

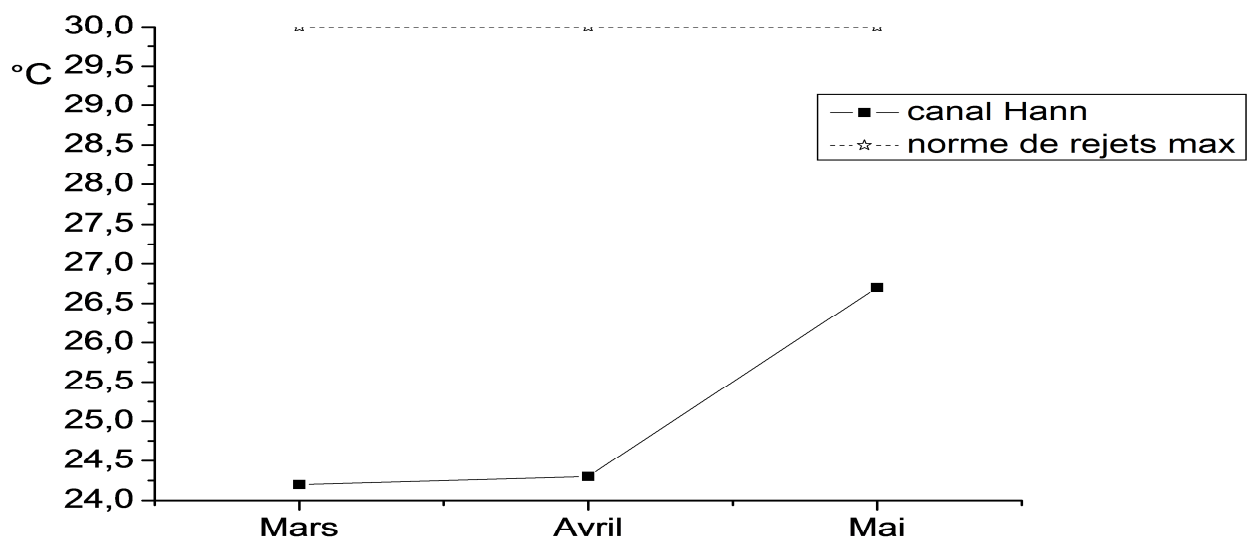

Figure 3: Mesures de la température dans les eaux usées en fonction du temps. 


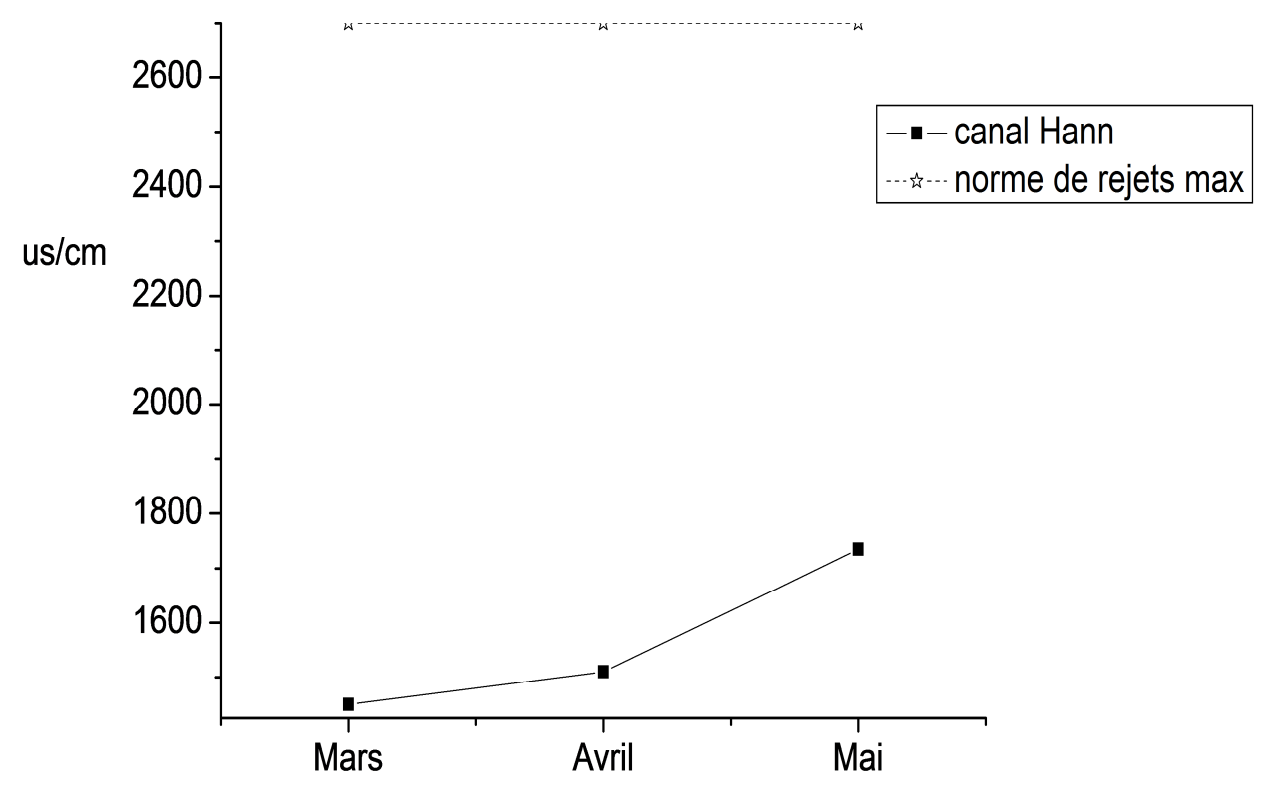

Figure 4: Mesures de la conductivité dans les eaux usées en fonction du temps.

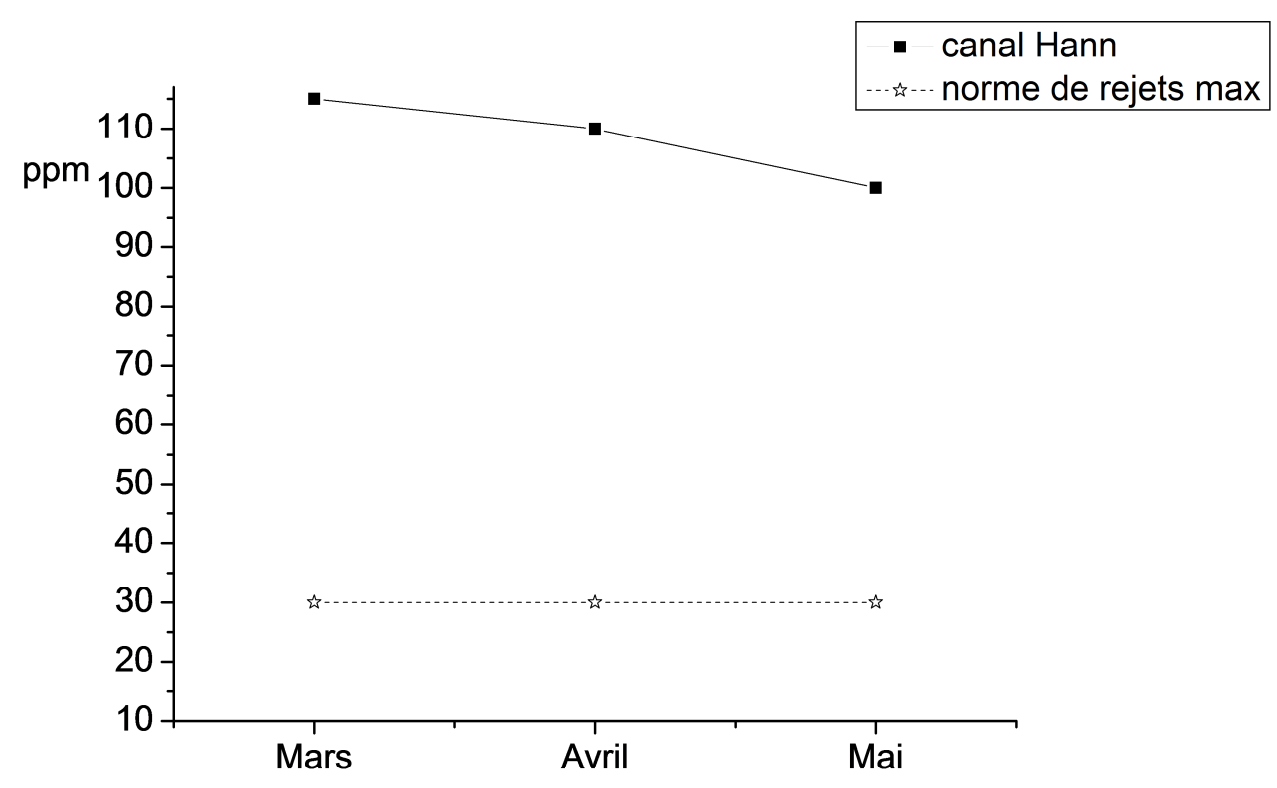

Figure 5: Evolution de la teneur de l'ammonium dans les eaux usées en fonction du temps. 


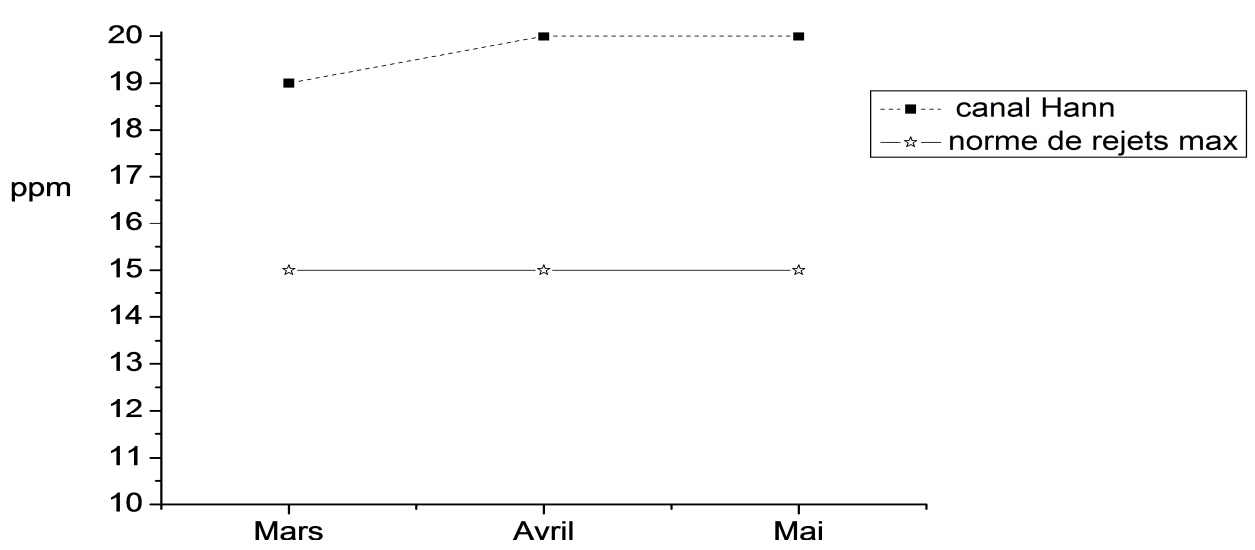

Figure 6: Evolution de la teneur du potassium dans les eaux usées en fonction du temps.

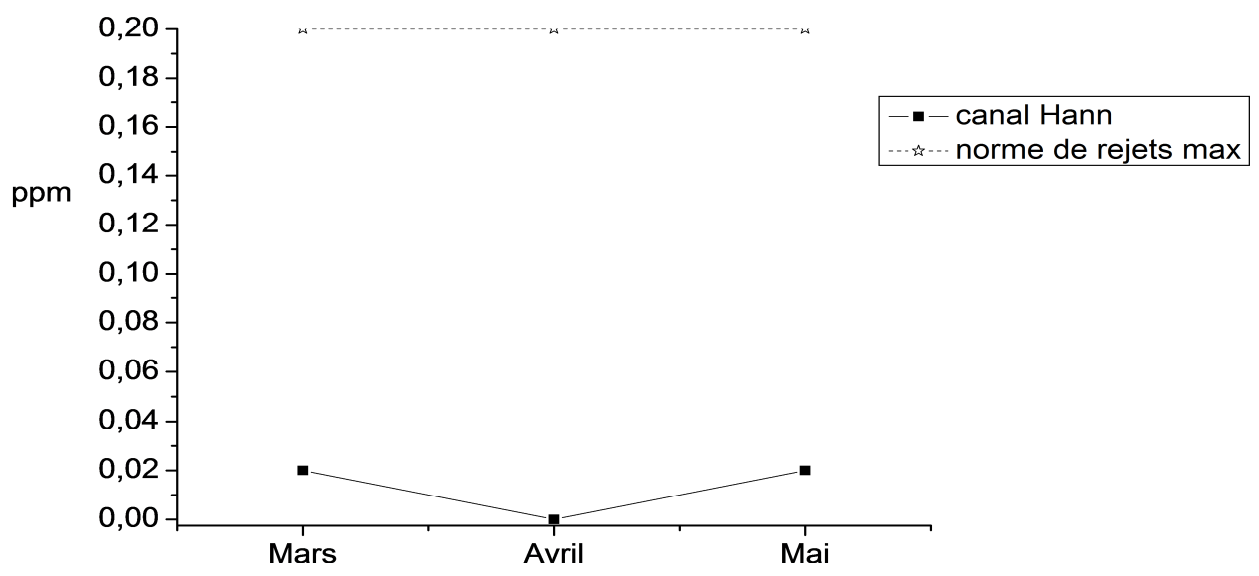

Figure 7:Evolution de la teneur du chrome dans les eaux usées en fonction du temps.

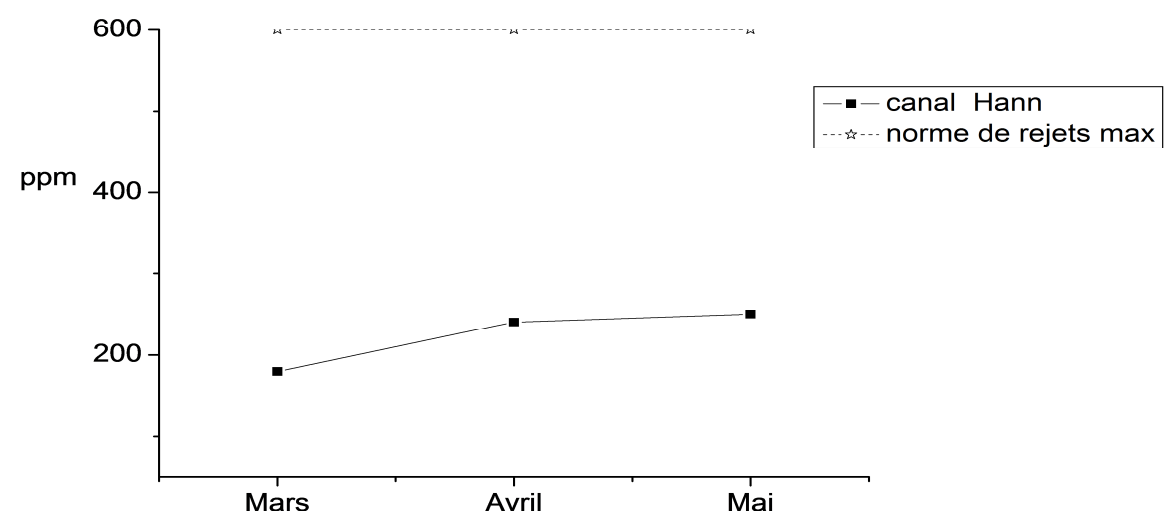

Figure 8: Evolution de la teneur des chlorures dans les eaux usées en fonction du temps. 


\section{DISCUSSION}

Le potentiel d'hydrogène d'une eau représente son acidité ou son alcalinité. Les résultats montrent que le $\mathrm{pH}$ est légèrement acide pour le canal de Hann. En effet, il oscille entre 6,88 et 6,98 . Par conséquent ces eaux ont des valeurs de $\mathrm{pH}$ qui concordent à la norme de rejets des eaux usées au Sénégal (Mouhamed et al., 2012). Elles permettent aussi un développement normal de la faune et de la flore qui exige des valeurs de $\mathrm{pH}$ comprises entre 5 et 9 (Blinda, 2007).

La température constitue une caractéristique physique importante. Elle joue un rôle dans la solubilité des sels et surtout des gaz, dans la détermination du $\mathrm{pH}$ pour la connaissance de l'origine de l'eau et des mélanges éventuels. Les résultats ont aussi montré que les températures sont légèrement variables d'un mois à un autre. Cette faible variation est due à la période d'analyses et à la température de la région. Les mesures oscillent entre 24,2 et $26,7^{\circ} \mathrm{C}$ correspondant aux normes de températures, dans les eaux de rejets, régies par la législation sénégalaise.

La conductivité traduit le degré de minéralisation des eaux et renseigne sur le taux de salinité. Elle permet aussi d'apprécier la quantité de sels dissous dans l'eau (Rodier et al., 1996). Les résultats de mesures varient entre 1450 et $1735 \mu \mathrm{S} / \mathrm{Cm}$. Ces valeurs sont, cependant, inférieures à $2700 \mu \mathrm{S} / \mathrm{Cm}$, considérée comme une valeur limite de rejets directs dans le milieu récepteur (Youssef et al., 2015). Par contre la minéralisation de ces eaux est élevée car la conductivité est supérieure à $1000 \mu \mathrm{S} / \mathrm{Cm}$ (Ahoudi et al., 2015).

L'ammonium est présent dans les eaux habituellement par un processus de dégradation incomplète de la matière organique. Il se transforme assez rapidement en nitrites et nitrates par oxydation. L'analyse de la Figure 5 montre que les teneurs en ammonium dans les eaux usées dépassent la valeur $30 \mathrm{ppm}$ qui correspond à la norme de rejets dans le milieu récepteur et à l'irrigation (Chafia et al., 2013). Certaines concentrations dépassent $100 \mathrm{ppm}$. La présence de ces ions peut être attribuée aux eaux de ruissellements urbains, industrielles ou domestiques qui contiennent de l'urine, potentielle source d'ammonium (Bonté et al., 2008).

Le potassium est un élément essentiel pour la croissance des plantes. On le trouve dans la plupart des sols et dans divers composés. Les concentrations en potassium enregistrées dans les eaux usées oscillent entre 19 et $20 \mathrm{ppm}$ (Figure 6). Ces teneurs dépassent légèrement $15 \mathrm{ppm}$, qui représentent la concentration normale de rejets du potassium dans le milieu récepteur.

La présence du chrome est le plus souvent liée aux rejets industriels provenant des ateliers de galvanoplastie, de tannage etc. (Sepher et al., 2005). Il est hautement toxique et s'accumule principalement dans les poumons. Il pénètre les cellules à travers le système de transport des ions. L'analyse de la Figure 7 montre que les concentrations en chrome 6 sont très faibles, à peu près 0,02 ppm et il n'est pas détecté en avril. Ces concentrations obtenues sont, cependant, inférieures à la valeur limite de rejets du chrome (VI) (0,2 ppm) dans le milieu récepteur (Mouhamed et al., 2012).

Les chlorures $\mathrm{Cl}^{-}$sont des ions très abondant dans l'environnement. Ils sont présents dans l'eau, le sol, les roches, ainsi que dans de nombreux aliments. Les teneurs en chlorures enregistrées dans notre étude varient entre 180 et 250 ppm (Figure 8). Ces 
concentrations ne dépassent pas la norme de rejets directs $(600 \mathrm{ppm})$ d'eaux usées dans le milieu récepteur (Menyakhlef et al., 2007).

Les phosphates sont des composants naturels des organismes vivants. Ils sont présents dans les excréments humains ainsi que dans les produits de lavage comme les poudres à lessiver et les produits de vaisselle. On les retrouve dans les eaux de surface par le biais des eaux usées. Les concentrations trouvées après analyse, varient entre 40 et 48 ppm (Figure 9). Les valeurs obtenues dépassent la limite de rejets $(10 \mathrm{ppm})$ autorisée dans le milieu récepteur (Belghyti et al., 2008). Ces fortes teneurs en phosphates peuvent être dues aux activités domestiques et aux rejets d'eaux industrielles très riches en phosphore.

Les sulfates ont une toxicité aiguë très faible. Par ailleurs, les teneurs élevées dans l'eau naturelle entrainent des nuisances d'ordre organoleptiques et sanitaires. En effet, ils provoquent des troubles gastro-intestinaux (en particulier chez les enfants) et peuvent donner un goût désagréable (Moussa Moumouni. 2005). Les résultats montrent des teneurs variant entre 21 et $30 \mathrm{ppm}$ (Figure 10). Ces valeurs sont, cependant, nettement inférieures à la limite $(400 \mathrm{ppm})$ de rejets d'eaux usées dans le milieu récepteur (Aboulhassan et al., 2008).

Les fluorures sont libérés dans l'environnement par des phénomènes naturels tels que la pluie, l'érosion, l'activité volcanique et des aérosols des eaux marines (Symonds et al., 1988). L'analyse de la Figure 11 nous renseigne que les concentrations en fluorures sont très faibles et varient entre 0,9 et $1,2 \mathrm{ppm}$. Ces valeurs sont négligeables devant la limite $(15 \mathrm{ppm})$ de rejets directs d'eaux usées dans le milieu récepteur (Zegaoula et al., 2014).

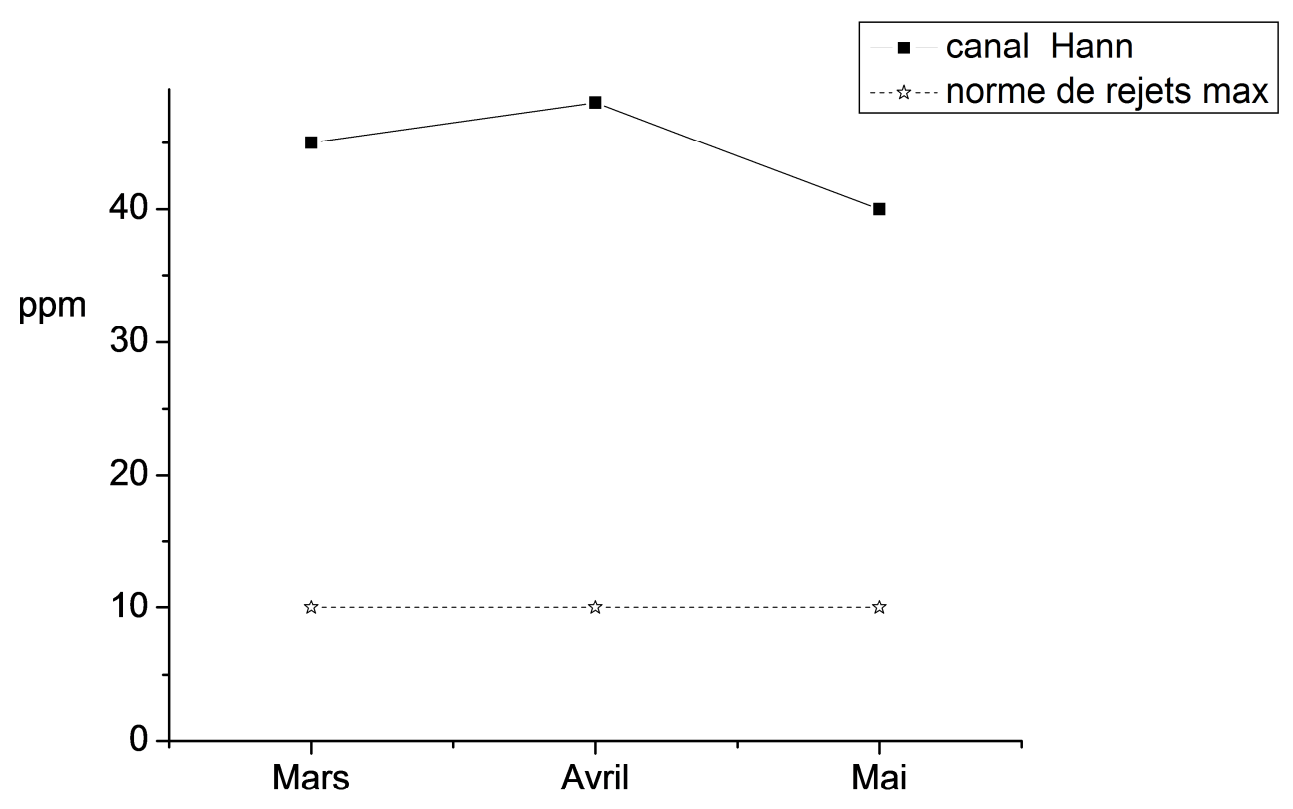

Figure 9: Evolution de la teneur du phosphate dans les eaux usées en fonction du temps. 


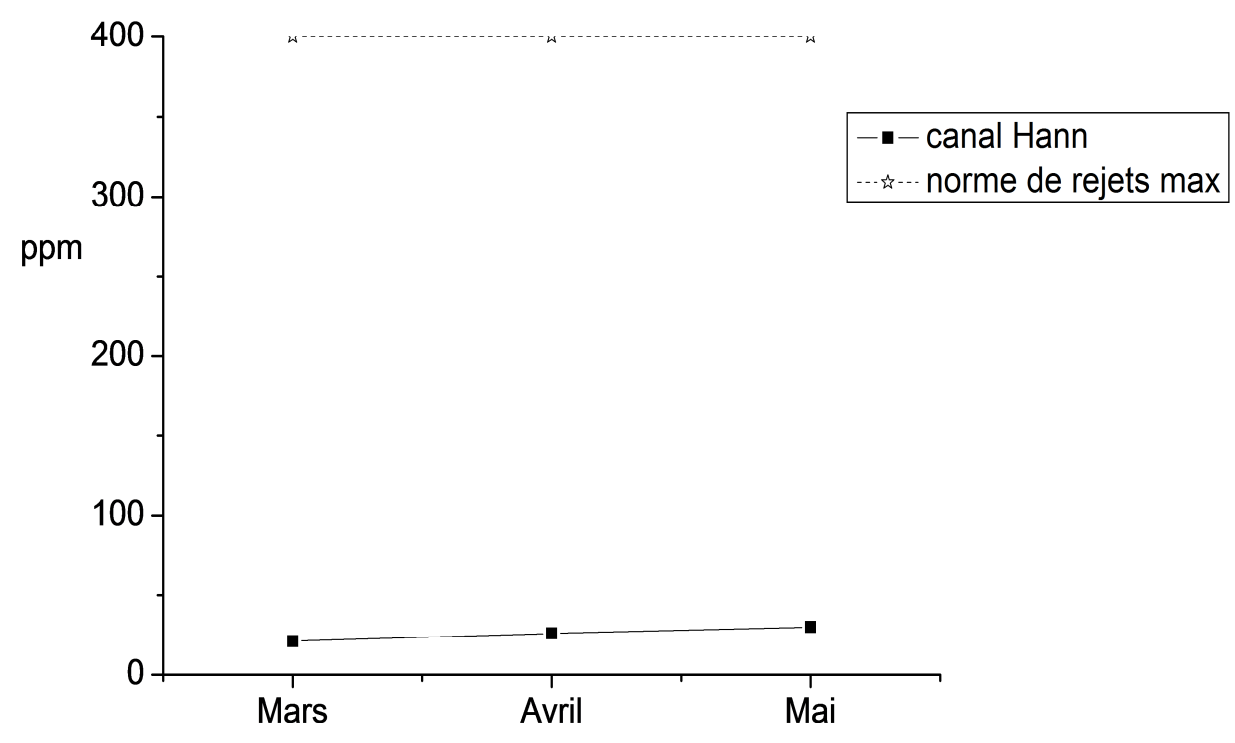

Figure 10: Evolution de la teneur des sulfates dans les eaux usées en fonction du temps.

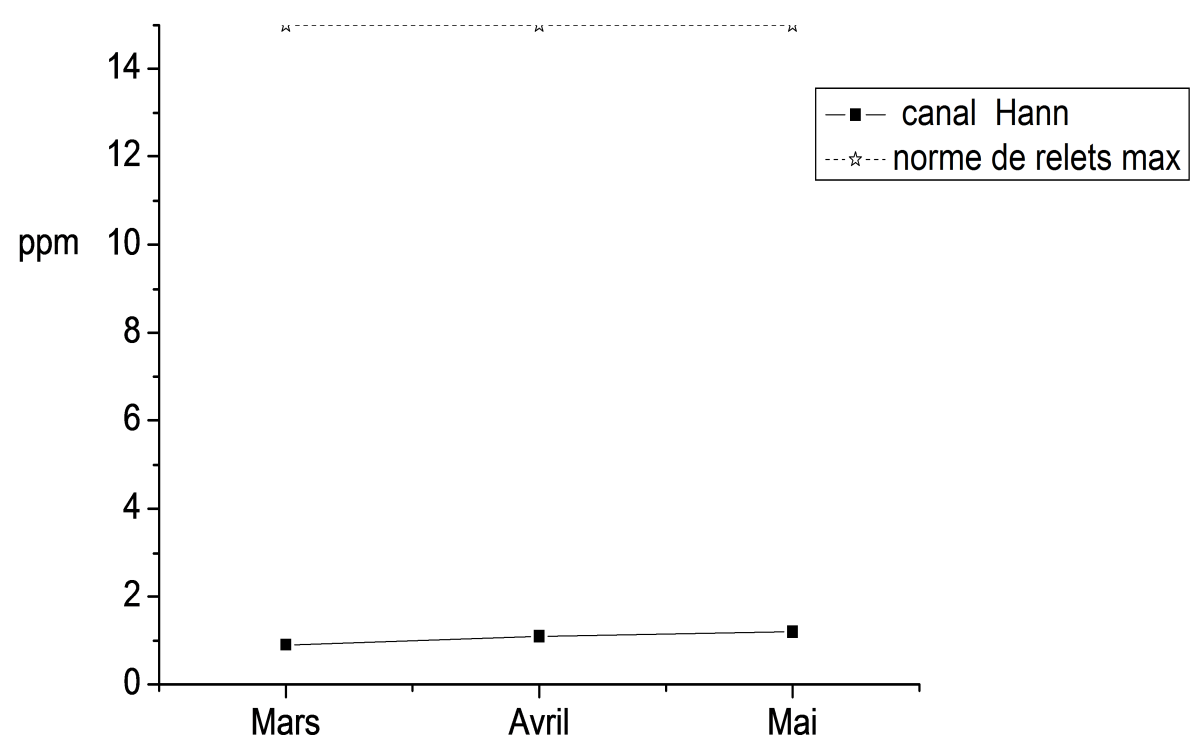

Figure 11: Evolution de la teneur des fluorures dans les eaux usées en fonction du temps. 


\section{Conclusion}

La qualité des eaux de mer de la région de Dakar dépend de la nature des eaux de rejets déversées directement sans aucun traitement préalable. Les résultats de mesures ont révélé la présence de chrome dans les eaux usées déversées directement dans la mer. Toutefois les teneurs trouvées répondent aux normes de rejets établies par la communauté scientifique, à l'exception des ions ammonium, potassium et phosphate. Ces ions ont des teneurs qui dépassent nettement les normes établies par la législation. Ainsi, l'installation de stations d'épurations et un suivi temporel d'analyse des eaux usées sont nécessaires pour préserver les côtes dakaroises.

\section{COMPETING INTERESTS}

The authors declare that they have no competing interests.

\section{CONTRIBUTIONS DES AUTEURS}

Les travaux ont été effectués au Laboratoire de Chimie Physique Organique et d'Analyses Environnement (LCPOAE) du Département de Chimie de l'Université Cheikh Anta Diop de Dakar, dirigé par le AD. L'encadrement de l'équipe de recherche est assuré par MN qui a élaboré le sujet et supervisé le travail. ID est l'investigateur principal. SD a participé à l'échantillonnage et à la préparation des solutions. $\mathrm{BN}$ a contribué à la réalisation des mesures.

\section{REMERCIEMENTS}

Les auteurs remercient Macherey Nagel qui a offert le spectrophotomètre et les kits de standards, et Monsieur Paul Nkeng de l’Université de Strasbourg.

\section{REFERENCES}

Aboulhassan MA, Souabi S, Yaacoubi A, Zaim N, Bouthir FZ. 2008. Les effluents de tannerie caracterisation et impact sur le milieu marin. Journal of Water Science, 21: 463-473.

Ahoudi H, Gnandi K, Tanouayi G, OuroSama K. 2015. Caractérisation physico-chimique et état de pollution par les éléments traces métalliques des eaux souterraines de Lomé : cas du quartier Agoe Zongo. Larhyss Journal, 24: 41-56.

Belghyti D, El Kharrim Kh, Raweh S, Sylla I, Benyakhef M. 2008. Etude physicochimique des eaux usées brutes de l'abattoir municipal de Kénitra (Maroc) en vue de la mise en œuvre d'un traitement convenable. Sud Science et Technologie, 16: 36-43.

Benyakhlef M, Naji S, Belghyti D. 2007. Caractérisation des rejets liquides d'une conserverie de poissons. Bull. Soc. Pharm., 146 : 225-234.

Berho C. 2008. Procédures d'échantillonnage des eaux souterraines en vue d'une analyse microbiologie. Etat de l'Art. Rapport final BRGM/RP-56405-FR, $26 \mathrm{p}$.

Blinda M. 2007. Pollution tellurique du littoral nord-ouest du Maroc entre Tanger et Tétouan: caractérisation, impact sur l'environnement et proposition de solutions. Thèse de doctorat, université Mohamed V, Faculté des Sciences, Rabat, pp. 194.

Bonté SL, Pons M, Potier O, Rocklin P. 2008. Relation between conductivity and ion content in urban wastewater. Journal of Water Science, 21(4): 429-438.

Chafia H, Abdelaziz B, Mohammed H. 2013. Caractérisation des rejets liquides d'une unité de réparation navale à Agadir. Revue Internationale d'Héliotechnique, 45: 29-36. 
Ezziane S. 2007. Traitement des eaux de rejets, le Mémoire présenté pour obtenir le diplôme de Magister, Université HASSIBA BEN BOUALI de CHLEF, 186p.

Mc Kinney ML. 2002. Urbaization, biodiversity and conservation. Biosci., 52: $883-890$.

Mohammed B, Abdelhamid B, Mohammed F. 2012. Etude de la qualité physicochimique des eaux usées de cinq villes de la région de la Chaouia-Ouardigha (Maroc). Bulletin de l'Institut Scientifique, Rabat, section Sciences de la Vie, 34(2): 145-150.

Moussa moumouni et al. 2005. Les eaux résiduaires des tanneries et des teintureries: Caractéristiques physicochimiques, bactériologiques et impact sur les eaux de surface et les eaux souterraines. Thèse de doctorat, université de Bamako, faculté de Médecine, de Pharmacie et d'odontostomalogie, p 52.
Rodier J, Bazin C, Broutin JP, Champsaur H, Rodier L. 1996. L'Analyse de l'Eau (8̀̀ édition). Dunod : Paris; 1384 p.

Sepher MN, Nasseri S, Assadi MM, Yaghmalan K. 2005. Chromium bioremoval from tannery industries effluent by AspergillusOryzae. Iran. J. Environ. Health, Sci. Eng., 2: 273-279.

Symonds RB, Rose WI, Reed MH. 1988. Contribution of $\mathrm{Cl}^{-}$and $\mathrm{F}^{-}$bearing gases to the atmosphere by volcanoes. Nature, 334: 415-418.

Youssef Azami I, Ali A, Saad A, Hajar D, Khadija E, Driss B. 2015. Caractérisation physico-chimique des eaux usées de la ville d'Azilal-Maroc. International journal of Innovation and Applied Studies, 11: 556-566.

Zegaoula W, Khellaf N. 2014. Evaluation du degré de pollution des rejets liquides et atmosphériques du complexe fertialAnnaba (Algérie). Larhyss Journal, 18 : 77-91. 